\title{
The Diagnostic Value of SCUBE1 in Unstable Angina Pectoris Patients
}

\author{
Abuzer Özkan', Ertan Sönmez², Serdar Özdemir ${ }^{3}$,̈mer Faruk Özer ${ }^{4}$, Nasifov Muharrem ${ }^{5}$, Bedia Gülen², Muhammed Keskin ${ }^{6}$ \\ ${ }^{1}$ Clinic of Emergency Medicine, Bağcılar Training and Research Hospital, İstanbul, Turkey \\ ${ }^{2}$ Department of Emergency Medicine, Bezmialem Vakıf University School of Medicine, İstanbul, Turkey \\ ${ }^{3}$ Clinic of Emergency Medicine, Ümraniye Training and Research Hospital, İstanbul, Turkey \\ ${ }^{4}$ Department of Biochemistry, Bezmialem Vakıf University School of Medicine, İstanbul, Turkey \\ ${ }^{5}$ Department of Cardiology, Bezmialem Vakıf University School of Medicine, İstanbul, Turkey \\ ${ }^{6} \mathrm{Clinic}$ of Cardiology, Siyami Ersek Thoracic and Cardiovascular Surgery Hospital, İstanbul, Turkey
}

\begin{abstract}
Aim: Signal peptide complement $\mathrm{C} 1 \mathrm{r} / \mathrm{C} 1 \mathrm{~s}$, Uegf, and Bmp1-epidermal growth factor-like domain-containing protein 1 (SCUBE1) has been used in research as a biomarker in acute coronary syndrome (ACS). The aim of this study is to determine if SCUBE1 and routine laboratory parameters are effective in distinguishing the patients presenting with clinical symptoms of unstable angina pectoris (USAP) from the patients who have non-ST-segment elevated myocardial infarction (NSTEMI) and non-cardiac chest pain patients (NCCP).
\end{abstract}

Materials and Methods: The study group consisted of patients having chest pain or those suspected of having an ACS complaint. The study consisted of 5 groups of 185 patients with USAP, NSTEMI, segment elevation myocardial infarction (STEMI), or NCCP, and a control group of 45 healthy patients (CG). The study was conducted according to the American Heart Association (AHA) guidelines.

Results: There was no statistical difference between the patient groups and CG in terms of SCUBE1 ( $p=0.650$ ). The Troponin I value was significantly lower in the USAP group than the STEMI and NSTEMI groups $(p<0.001)$. There was no difference among the sub-groups of USAP in terms of the SCUBE1, GENSINI, and the GRACE scores $(p=0.485,0.932,0.585$, respectively).

Conclusion: SCUBE1 was not diagnostic for USAP, NSTEMI, and STEMI. Further studies to understand the value of SCUBE1 in ACS are needed.

Keywords: Acute coronary syndrome, unstable angina pectoris, SCUBE1

\section{Introduction}

Acute coronary syndrome (ACS) develops when there is a misbalance between supplying oxygen to the myocardium and the myocardium's need for oxygen. Unstable angina pectoris (USAP) is attributed to the group of patients whose electrocardiography (ECG) does not show any biological symptoms of chest pain, or the equivalent of ACS, and whose biological indicator does not increase (1). The patients presenting with symptoms of chest pain or symptoms relating to ACS who do not have persistent ST-segment elevation but have pathological findings in electrocardiography (ECG), and whose Troponin I values are high, are defined as non-ST-segment elevated myocardial infarction (NSTEMI) (2).
The evaluation of patients presenting with chest pain in the emergency and outpatient environment are completed using the combination of biochemical examinations that reflect clinical history, examination findings, ECG, and myocardial ischemia (3). As ischemic biomarkers, sensitive cardiac Troponin I assays (with an ability of detection below the $99^{\text {th }}$ percent of a reference population and improved precision) have recently become available in clinical practice (4-7). Many types of parameters are used to distinguish USAP patients from non-cardiac chest pain patients (NCCP) patients (8). Even though the specificity and the sensitivity of Troponin I are high in myocardial infarction (MI), it is not enough to distinguish the USAP patients from the NCCP patients; and in order to detect it in the blood, time is needed. Therefore, new and more convenient bio-

Correspondence to: Ertan Sönmez e-mail: ertansnmz@gmail.com

Received: 21.07.2016 Accepted: 29.09.2016

(C) Copyright 2016 by Emergency Physicians Association of Turkey - Available online at www.eajem.com DOI: 10.5152/eajem.2016.83997 
chemical parameters are needed. SCUBE1 has been recently used as an early indicator for the diagnosis of ACS (9).

The molecules of SCUBE1 are stored inside the alpha granules in the inactive platelets. After the activation by thrombin, it is translocated to the surface of the platelet, secreted as small soluble forms, and incorporated into the thrombus. The accumulation of SCUBE1 is detected in human beings as immunohistochemistry in the sub-endothelial matrix of advanced atherosclerotic lesions (4). Our primary goal in this study was to evaluate whether SCUBE1 and routine laboratory parameters are effective in differentiating patients presenting with the clinical symptoms of USAP from the ones with NSTEMI and NCCP. Our second goal was to evaluate whether SCUBE1 would be a predictive biochemical indicator of the prevalence and severity of coronary artery disease in all patients.

\section{Materials and Methods}

\section{Study design}

This study was approved by our institutional ethical review boards. Written informed consent was obtained from the participants. The investigation conforms to the principles outlined in the Declaration of Helsinki.

\section{Study population}

In this study, 220 patients who had chest pain, sweating that gave the impression of ACS, shortness of breath, nausea, stomach ache, fainting problems that are equal to angina symptoms, and 45 healthy volunteers participated. Thirty-five of the participants were omitted because of their refusal of treatment, lack of data in their files, or laboratory mistakes. The study groups were designed according to AHA criteria (2). Patients were classified into four groups: USAP $(n=44)$ because there was a myocardial ischemia without myocardial necrosis; NSTEMI $(n=50)$ because they were associated with elevated myocardial necrosis markers and without any elevation in ST-segments in the ECG; segment elevation myocardial infarction (STEMI) $(n=45)$ because they had acute chest pain and/or persistent (>20 min) ST-segment elevation and elevated necrosis markers; and $\operatorname{NCCP}(n=46)$. The verification of the ACS diagnosis was done by angiography. In total, 230 patients were enrolled (including the control group (CG) consisting of 45 healthy individuals). USAP and NSTEMI groups were classified into three groups according to their GRACE scores: 1-108=low, 109-140=average, and 141-372=high. The GENSI$\mathrm{NI}$ score was classified into two sub-categories: $0-19=$ mild, $>20=$ serious. Blood samples were collected from the patients on admission to the emergency department for their complaints. We studied the SCUBE1, Troponin I, and creatine kinase-MB (CK-MB). A power analysis $(1-\beta=0.9, a=0.05)$ determined that a NCCP size of 16 patients and a CG size of 13 patients were sufficient to stratify this patient cohort; 91 patients in total (NCCP: 46 and CG: 45) were enrolled.

\section{Exclusion criteria}

People under 18 years of age, people who had problems in the last one month such as pulmonary embolism, ischemic stroke, deep vein thrombosis, peripheral arterial embolism, pneumonitis, ACS, transient ischemic attack, pulmonary edema, hemorrhagic stroke, serious valvular heart disease, myocarditis, endocarditis, coronary artery bypass grafting, and coronary percutaneous cases were excluded from the study. Renal impairments with serum creatinine levels higher than 2.5, hepatitis with elevated liver enzymes, cancer, hematologic diseases, and rheumatology diseases were also excluded from the study. Lastly, pregnant or recently postpartum females were excluded.

\section{Preparations of samples and measurements}

Blood samples were collected from the patients on admission. For SCUBE1 measurements, blood samples were kept 10-20 minutes, and centrifuged at $3000 \mathrm{cycle} / \mathrm{min}$ for ten minutes and then kept at -30 degree Celsius in a freezer. Before the measurements, the samples reached room temperature. An ELISA study was conducted with SCUBE1 (Human Signal Peptide, Cub and Uegf-like Domain Containing Protein 1, Elisa Kit, Bioassay Technology Laboratory, Catalogue Number: E3142hu. Crystal Day Biotech Co. Ltd., China). The test results were compared with other patient parameters.

\section{Statistical analysis}

The data obtained from the study was calculated through the program IBM Statistical Package for Social Sciences version 20 (IBM SPSS Statistics; Armonk, NY, USA). Quantitative data were expressed as median, min-max; and qualitative values were expressed as a percentage. Chi-square analysis was performed for nominal data. End group analysis was performed. The Kolmogorov-Smirnov test was used to evaluate whether the numerical variables were normally distributed or not. Groups and subgroups did not show normal distribution. Accordingly, a Kruskal-Wallis analysis was used, and statistically significant differences were seen between the patient subgroups $(p<0.01)$. A Bonferroni corrected Mann-Whitney $U$ test was used to assess the differences $(p=0.05 / 10=0.005)$. Spearman correlation analysis was performed to evaluate the relationship between SCUBE1 levels and variables. Calculated $p$ values less than 0.05 were considered statistically significant.

\section{Results}

There was no correlation between SCUBE1 values and age. There was no significant difference between the patients and the CG groups in SCUBE1 values $(p=0.65)$ (Figure 1). Troponin I values were different among the patients and the CG groups $(p<0.001)$. Troponin I levels in the USAP, NSTEMI and STEMI groups were significantly higher than the NCCP group $(p<0.001)$. Troponin I levels in the USAP group were significantly lower than the STEMI and NSTEMI groups $(p<0.001)$ (Figure 2).

In NSTEMI and STEMI groups, CK-MB values were significantly higher than the NCCP and CG groups $(p<0.001)$. Routine hematologic parameters were calculated (Table 1 ).

In terms of the risk factors for ACS, 40 of the 44 USAP patients (52.3\%) had chronic atherosclerosis disease in their previous patient history (Table 2). There was no difference among the USAP middle-low and high-risk sub groups in terms of SCUBE1, GENSINI, and GRACE scores ( $p=0.481,0.932$, and 0.585 , respectively) (Table 3 ). There were no differences for SCUBE1 values between the low and high-risk groups in the GENSINI, which were calculated according to the angiography results of the USAP, NSTEMI, and STEMI groups $(p=0.59)$. In the values of GRACE's inside-hospital risk scoring, there was no difference among the three groups, which were categorized as low, middle, and high risk for the values of SCUBE1 $(p=0.370)$. There were differences among the groups that were categorized as 


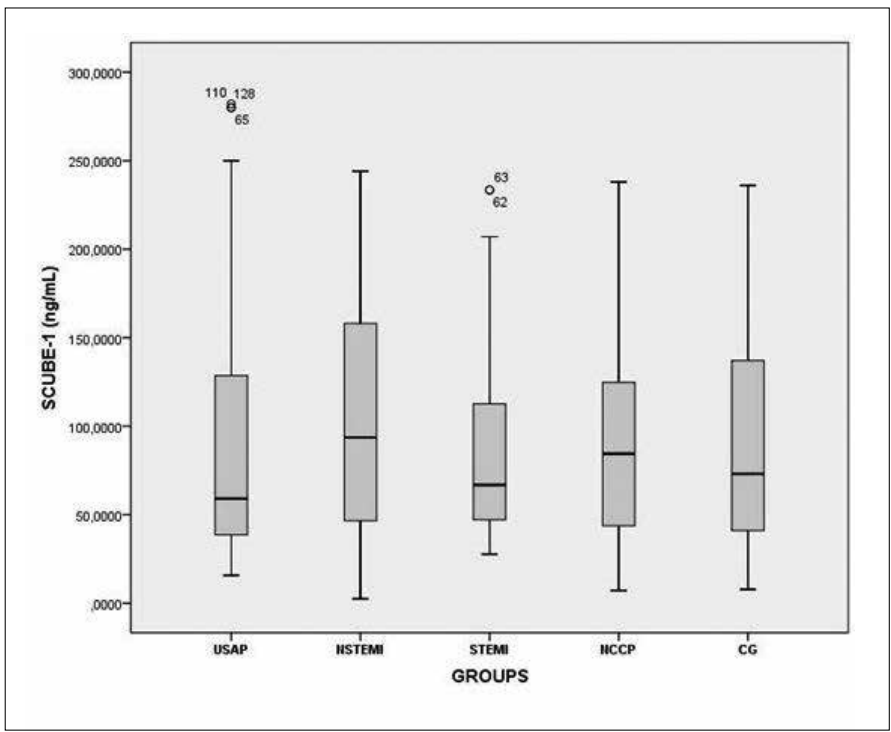

Figure 1. SCUBE1 values distribution according to groups

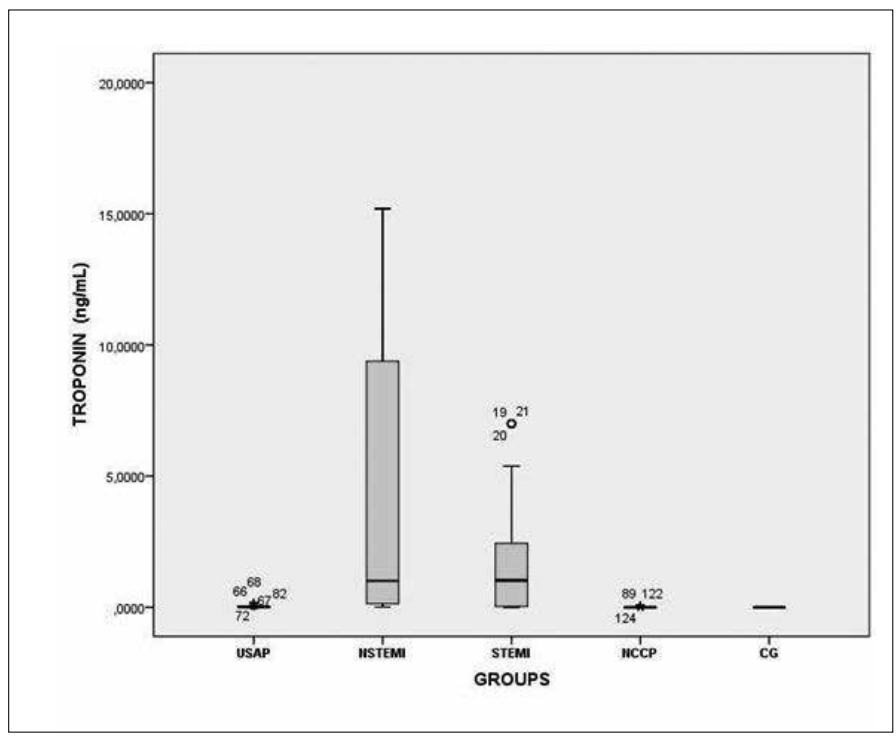

Figure 2. Troponin I values according to groups

Table 1. Statistical significance of variables according to study groups

\begin{tabular}{|c|c|c|c|c|c|c|}
\hline & USAP & NSTEMI & STEMI & NCCP & CG & $\mathbf{P}^{*}$ \\
\hline SCUBE1, ng/mL & $93,15-282$ & $103,2-244$ & $88,27-233$ & $90,7-238$ & $93,7-236^{b}$ & 0.65 \\
\hline Age, years & $58,32-84^{\mathrm{a}, \mathrm{c}}$ & $62,32-87^{a, b, e}$ & $63,36-89^{a, b, e}$ & $49,18-84^{a, c, d}$ & $35,17-71^{b, c, d, e}$ & $<0.001$ \\
\hline Troponin I, ng/mL & $0.02,0-0.1^{\mathrm{a}, \mathrm{c}, \mathrm{d}, \mathrm{e}}$ & $4,0-15,2^{a, b, e}$ & $2,0-7^{a, b, e}$ & $0,0-0.04^{b, c, d}$ & $0,0-0^{b, c, d}$ & $<0.001$ \\
\hline CK-MB (ng/dL) & $20,11-43^{e}$ & $30,1-76^{a, e}$ & $31,4-70^{a, e}$ & $14,1-27$ & $15,3-24^{c, d}$ & $<0.001$ \\
\hline GENSINI score & $19,0-116^{\mathrm{a}, \mathrm{d}, \mathrm{e}}$ & $330-139^{a, e}$ & $48,0-147^{\mathrm{a}, \mathrm{b}, \mathrm{e}}$ & $0,0-0^{b, c, d}$ & $0,0-0^{b, c, d}$ & $<0.001$ \\
\hline GRACE risk score & $90,47-152^{\mathrm{a}, \mathrm{c}, \mathrm{d}, \mathrm{e}}$ & $110,41-168^{a, b, d, e}$ & $142,94-202^{a, b, c, e}$ & $0,0-0^{b, c, d}$ & $0,0-0^{b, c, d}$ & $<0.001$ \\
\hline MPV, fL & $10,8-12^{a, c, d, e}$ & $9,5-12^{a, b}$ & $9,7-12^{a, b, e}$ & $8,6-11^{b, d}$ & $7,6-10^{b, c, d}$ & $<0.001$ \\
\hline Hemoglobin, g/dL & $13,7-17$ & $13,7-16$ & $13,9-17^{e}$ & $12,12-15^{\mathrm{a}}$ & $13,12-14^{\mathrm{e}}$ & 0.011 \\
\hline Neutrophils, & $58,43-82^{a}$ & $62,22-88^{a}$ & $62,46-95^{a}$ & $54,35-77$ & $52,49-65^{b, c, d}$ & $<0.001$ \\
\hline Lymphocytes, & $31,10-41^{a}$ & $27,5-46^{a}$ & $28,4-42^{\mathrm{a}}$ & $35,6-50^{a}$ & $36,26-39^{b, c, d, e}$ & $<0.001$ \\
\hline $\mathrm{WBC}, 10^{3} / \mu \mathrm{L}$ & $7.2,4.5-11.4$ & $9.2,4.7-15.7$ & $11.2,5.3-21.1$ & $8.4,4.3-14.2$ & $7.7,5.0-9.9$ & 0.523 \\
\hline $\mathrm{PCT} n \mathrm{~g} / \mathrm{mL}$ & $0.2,0-0.4^{a, c, e}$ & $0.2,0-0.3^{b}$ & $0.2,0.1-0.4$ & $0.19,0.16-0.39$ & $0.19,0.16-0.39^{b}$ & 0.003 \\
\hline PLT, $10^{3} / \mathrm{mL}$ & $251,120-451$ & $238,118-317$ & $239,119-464$ & $221,171-456$ & $263,172-456$ & 0.498 \\
\hline Creatinine, $\mathrm{mg} / \mathrm{dL}$ & $0.8,0.4-1.4^{d}$ & $0.8,0.5-4.2^{\mathrm{a}}$ & $0.8,0.5-2.1^{a, b}$ & $0.7,0.6-1.3$ & $0.6,0.6-0.9^{c, d}$ & $<0.001$ \\
\hline RDW fl & $42,16-63$ & $42,34-48$ & $41,38-48$ & $40,37-47$ & $42,34-48$ & 0.213 \\
\hline PDW fl & $12,9-21^{\mathrm{a}, \mathrm{c}, \mathrm{d}, \mathrm{e}}$ & $17,10-20^{\mathrm{a}, \mathrm{b}, \mathrm{d}, \mathrm{e}}$ & $18,10-21^{b, c}$ & $18,17-21^{b, c}$ & $18,16-19^{\mathrm{b}, \mathrm{c}}$ & $<0.001$ \\
\hline AST, 0-45 IU/L & $20,13-60^{d}$ & $26,5-131^{\mathrm{a}, \mathrm{e}}$ & $32,12-133^{a, b, e}$ & $18,8-47^{c, d}$ & $19,14-21^{\mathrm{c}, \mathrm{d}}$ & $<0.001$ \\
\hline ALT, 0-45 IU/L & $22,11-66^{a, e}$ & $17,8-60$ & $23,8-173^{a, e}$ & $17,10-29^{b, d}$ & $17,8-19^{b, d}$ & $<0.001$ \\
\hline \multicolumn{7}{|c|}{$\begin{array}{l}\text { USAP: unstable angina pectoris; NSTEMI: non-ST-segment elevated myocardial infarction; STEMI: segment elevation myocardial infarction; NCCP: non-cardiac chest } \\
\text { pain patients; CG: control group of } 45 \text { healthy patients; CK-MB: creatine kinase MB; WBC: white blood cell; PLT: platelet; PDW: platelet distribution width; RDW: red cel } \\
\text { distribution width; MPV: mean platelet volume; PCT: plateletcrit; AST: aspartate aminotransferase; ALT: alanine aminotransferase } \\
\text { Data are presented as median, min-max values } \\
\text { *Kruskal-Wallis H Test } \\
\text { ap }<0.005 \text { Control (Bonferoni corrected Mann-Whitney U Test) } \\
\text { b } p<0.005 \text { USAP (Bonferoni corrected Mann-Whitney U Test) } \\
\text { c } p<0.005 \text { NSTEMI (Bonferoni corrected Mann-Whitney U Test) } \\
\text { d } p<0.005 \text { STEMI (Bonferoni corrected Mann-Whitney U Test) } \\
\text { ep }<0.005 \text { NCCP (Bonferoni corrected Mann-Whitney U Test) }\end{array}$} \\
\hline
\end{tabular}


Table 2. The frequency rate of diseases among the groups

\begin{tabular}{|l|c|c|c|c|c|}
\hline & USAP, $\mathbf{n = 4 4}$ & NSTEMI, $\mathbf{n = 5 0}$ & STEMI, $\mathbf{n = 4 5}$ & NCCP, $\mathbf{n = 4 6}$ & $\mathbf{C G ,} \mathbf{n = 4 5}$ \\
\hline CAD & $23,52.3 \%$ & $10,20.0 \%$ & $7,16.3 \%$ & $0,0.0 \%$ & $0,0.0 \%$ \\
\hline DM & $18,40.9 \%$ & $11,22.0 \%$ & $13,28.9 \%$ & $0,0.0 \%$ \\
\hline Smokers & $7,15.9 \%$ & $8,16.0 \%$ & $11,24.4 \%$ & $0,0.0 \%$ & $0,0.0 \%$ \\
\hline HL & $7,15.9 \%$ & $7,14.0 \%$ & $0,0.0 \%$ & $0,0.0 \%$ & $0,0.0 \%$ \\
\hline HT & $27,61.4 \%$ & $23,46.0 \%$ & $15,33.3 \%$ & $2,4.8 \%$ & $0,0.0 \%$ \\
\hline COPD & $1,2.3 \%$ & $1,2.0 \%$ & $8,17.8 \%$ & $0,0.0 \%$ & $0,0.0 \%$ \\
\hline
\end{tabular}

USAP: unstable angina pectoris; NSTEMI: non-ST-segment elevated myocardial infarction; STEMI: segment elevation myocardial infarction; NCCP: non-cardiac chest pain patients; CG: control group of 45 healthy patients; CAD: coronary artery disease, DM: diabetes mellitus, HL: hyperlipidemia, HT: hypertension, COPD: chronic obstructive pulmonary disease

Data presented as number (percentage)

${ }^{*}$ Chi-square test

Table 3. The scoring values of SCUBE1 in GENSINI and GRACE among the sub groups of USAP

\begin{tabular}{|l|l|c|c|c|}
\hline USAP & SCUBE1 & GENSINI & GRACE \\
\hline \multirow{4}{*}{ High risk } & $\mathrm{n}$ & 25 & 25 & 25 \\
\cline { 2 - 5 } & Average & 106 & 18 & 92 \\
\cline { 2 - 5 } & Standard deviation & 90 & 28 & 21 \\
\cline { 2 - 5 } & Median & 82 & 6 & 88 \\
\cline { 2 - 5 } & Lowest & 16 & 0 & 55 \\
\cline { 2 - 5 } & Highest & 351 & 116 & 152 \\
\hline Middle-low risk & $\mathrm{n}$ & 19 & 19 & 19 \\
\cline { 2 - 5 } & Average & 79 & 19 & 87 \\
\cline { 2 - 5 } & Standard deviation & 74 & 30 & 23 \\
\cline { 2 - 5 } & Median & 53 & 7 & 88 \\
\cline { 2 - 5 } & Lowest & 15 & 0 & 47 \\
\cline { 2 - 5 } & Highest & 282 & 100 & 142 \\
\cline { 2 - 5 } & p & 0.485 & 0.932 & 0.585 \\
\hline
\end{tabular}

USAP: unstable angina pectoris

low, middle, and high in calculations of the six-month risk GRACE scores $(p=0.022)$. The NSTEMI group had a higher six-month risk GRACE score than the USAP group.

\section{Discussion}

In our study, we aimed to find SCUBE1's efficacy in separating USAP and NSTEMI, which was already studied in various ischemic situations because ACS, ischemic stroke, and CAD showed positive results $(4,9)$ Also, we aimed to discover if SCUBE1 levels can differentiate the NCCP patients from the ACS patients. We compared some parameters and two ACS scorings with SCUBE1: Troponin I, CK-MB, and the degrees of GRACE and GENSINI scores.

Troponin I and CK-MB are used in the diagnosis of myocardial infarction as cardiac indicators all around the world. However, their roles are limited in USAP diagnosis and require time for diagnosis (10, 11). Before the start of myocardial necrosis in the early period when the coronary perfusion defects occur, novel indicators will have an important necessity in diagnosis and treatment.

The SCUBE1 protein was first reported in the field of inflammation. Initially, SCUBE1 was only thought to be secreted in endothelial cells (12). In the study they conducted, Karabacak et al. (13) revealed that there was a meaningful difference between the SCUBE1 rates of hypertensive crises patients who presented to emergency services in comparison with a healthy control group. It is thought that this result can be related to probable endothelium damage. Tu et al. (14) showed that SCUBE1 was secreted at a higher rate than thrombocytes. It is known that thrombocyte aggregation accounts for ACS and acute ischemic stroke. Studies have proved that SCUBE1 is secreted from an active thrombocyte surface (4). They showed that it is secreted from alpha granules following thrombocyte aggregation and SCUBE1 is contained in the human RNA as well. Moreover, SCUBE1 was detected in thrombus and atherosclerotic lesions that are rich in thrombocytes (15). In the study of Mentese et al. (16), it was stated that the reason for the higher SCUBE1 level found in a gastric cancer study can be related to high thrombosis existing in probable malignancies. It is not clearly understood how SCUBE1 functions in atherosclerotic plaques and thrombus or why it is secreted by active thrombocytes.

In their study, Dai et al. (4) showed that plasma SCUBE1 is measurable in plasma for about four days, and starts to rise within 6 hours after thrombocyte activation. However, the rapid rise of SCUBE1 was shown in the study by Turkmen et al (17), in which they found SCUBE 1 rises rapidly 2 hours after the start of acute mesentery ischemia. SCUBE1 was considerably higher in comparison to the control group in chronic CAD, ACS, and acute ischemic stroke (AIS). Nevertheless, they expressed it would not be accurate to establish a meaningful correlation with a single measurement of SCUBE1 in the control group (12). Peacock WF, from Ohio University, said that the study by Dai et al. (4) was a promising indicator; but he criticized the mistakes in methodology in the study (18). Sonmez, one of our authors, previously studied SCUBE1 in ACS patients. In that study, in terms of SCUBE1, there were significant differences between the NSTEMI and NCCP groups (8). But there were no significant differences between the CG group and the patients in terms of SCUBE1 in our study $(p=0.65)$.

Troponin I rates were found to be significantly higher in the NSTEMI and STEMI groups than in the NCCP group. Troponin I could 
distinguish NCCP patients from MI patients, and it was detected as significantly lower than the NSTEMI group in the USAP group. The parameters for Troponin I in our study were compatible with the literature (19). Francis et al found in their study, which was conducted with 710 patients, that CK-MB rates detected the ACS patients (20). CK-MB was high in ACS patients in our study as well. Considering the research, there emerged contradictory results in similar studies of SCUBE1. In our opinion, one of the reasons may be the studying techniques and kit differences. Another reason may be the blood sampling times. But in this, and the previous studies, blood sampling times were not classified in the patients. There might be other reasons as well. We are of the opinion that new research is needed to discuss this issue.

\section{Study limitations}

Blood sampling times were not classified for the patients. It might have affected the results. This study resulted as negative for SCUBE1 to diagnose any ACS. As far as we know, the kit that we used was different from the previously used kits. The lack of positive or negative findings for this kit was the limitation for efficient discussion. We did not classify the time between the patients' admission to the emergency department and the start of their complaints.

\section{Conclusion}

In our study, we found that SCUBE1 was not an effective marker for USAP and other ACS patients. No negative or positive correlations were detected between the values of SCUBE1 and the scorings of GENSINI and GRACE. The contradictory results were gathered from studies done with SCUBE1 and further studies are needed to enlighten this point.

Ethics Committee Approval: Authors declared that the research was conducted according to the principles of the World Medical Association Declaration of Helsinki "Ethical Principles for Medical Research Involving Human Subjects", (amended in October 2013).

Informed Consent: Written informed consent was obtained from participants who participated in this study.

Peer-review: Externally peer-reviewed.

Conflict of Interest: No conflict of interest was declared by the authors.

Financial Disclosure: The authors declared that this study has received no financial support.

\section{References}

1. Bassand J-P, Hamm CW, Ardissino D, Boersma E, Budaj A, Fernández-Avilés $F$, et al. Guidelines for the diagnosis and treatment of nonST-segment elevation acute coronary syndromes. Eur Heart J 2007; 28: 1598-660. [CrossRef]

2. Braunwald E, Antman EM, Beasley JW, Califf RM, Cheitlin MD, Hochman JS, et al. ACC/AHA guidelines for the management of patients with unstable angina and non-ST-segment elevation myocardial infarction: a report of the American College of Cardiology/American Heart Association Task Force on Practice Guidelines (Committee on the Management of Patients With Unstable Angina). J Am Coll Cardiol 2000; 36: 970-1062. [CrossRef]
3. Apple FS, Wu AH. Myocardial infarction redefined: role of cardiac troponin testing. Clin Chem 2001; 47: 377-9.

4. Dai DF, Thajeb P, Tu CF, Chiang FT, Chen CH, Yang RB, et al. Plasma concentration of SCUBE1, a novel platelet protein, is elevated in patients with acute coronary syndrome and ischemic stroke. J Am Coll Cardiol 2008; 51: 2173-80. [CrossRef]

5. Apple FS, Wu AH, Jaffe AS, Panteghini M, Christenson RH, Cannon CP, et al. National Academy of Clinical Biochemistry and IFCC Committee for Standardization of Markers of Cardiac Damage Laboratory Medicine practice guidelines: analytical issues for biomarkers of heart failure. Circulation 2007; 116: e95-8. [CrossRef]

6. Apple FS, Smith SW, Pearce LA, Ler R, Murakami MM. Use of the Centaur Tnl-Ultra assay for detection of myocardial infarction and adverse events in patients presenting with symptoms suggestive of acute coronary syndrome. Clin Chem 2008; 54: 723-8. [CrossRef]

7. Melanson SE, Morrow DA, Jarolim P. Earlier detection of myocardial injury in a preliminary evaluation using a new troponin I assay with improved sensitivity. Am J Clin Pathol 2007; 128: 282-6. [CrossRef]

8. Parkash O, Almas A, Hameed A, Islam M. Comparison of non cardiac chest pain (NCCP) and acute coronary syndrome (ACS) patients presenting to a tertiary care center. J Pak Med Assoc 2009; 59: 667-71.

9. Sonmez E, Turkdogan KA, Karabacak M, Civelek C, Yilmaz C, Ozer OF, et al. The diagnostic role of signal peptide-C1r/C1s, Uegf, and Bmp1-epidermal growth factor domain-containing protein 1 in non-ST-elevation acute coronary syndrome. Am J Emerg Med 2015; 33: 21-4. [CrossRef]

10. Giannitsis E, Becker M, Kurz K, Hess G, Zdunek D, Katus HA. High-sensitivity cardiac troponin $\mathrm{T}$ for early prediction of evolving non-ST-segment elevation myocardial infarction in patients with suspected acute coronary syndrome and negative troponin results on admission. Clin Chem 2010; 56: 642-50. [CrossRef]

11. Tonino PA, De Bruyne B, Pijls NH, Siebert U, Ikeno F, van't Veer M, et al. Fractional flow reserve versus angiography for guiding percutaneous coronary intervention. N Engl J Med 2009; 360: 213-24. [CrossRef]

12. Lindemann S, Gawaz M. SCUBE1--a new scoop in vascular biology? Cardiovasc Res 2006; 71: 414-5. [CrossRef]

13. Karabacak M, Dogan A, Turkdogan AK, Kapci M, Duman A, Akpinar O. Mean platelet volume is increased in patients with hypertensive crises. Platelets 2014; 25: 423-6. [CrossRef]

14. Tu CF, Yan YT, Wu SY, Djoko B, Tsai MT, Cheng CJ, et al. Domain and functional analysis of a novel platelet-endothelial cell surface protein, SCUBE1. J Biol Chem 2008; 283: 12478-88. [CrossRef]

15. Tu CF, Su YH, Huang YN, Tsai MT, Li LT, Chen YL, et al. Localization and characterization of a novel secreted protein SCUBE1 in human platelets. Cardiovasc Res 2006; 71: 486-95. [CrossRef]

16. Mentese A, Fidan E, Sumer AU, Karahan SC, Sonmez M, Altay DU, et al. Is SCUBE 1 a new biomarker for gastric cancer? Cancer Biomark 2012; 11: 191-5.

17. Turkmen S, Mentese S, Mentese A, Sumer AU, Saglam K, Yulug E, et al. The value of signal peptide-CUB-EGF domain-containing protein 1 and oxidative stress parameters in the diagnosis of acute mesenteric ischemia. Acad Emerg Med 2013; 20: 257-64. [CrossRef]

18. Peacock WF. Will SCUBE1 solve the ischemia marker deficit? J Am Coll Cardiol 2008; 51:2181-3. [CrossRef]

19. Jeremias A, Gibson CM. Narrative review: alternative causes for elevated cardiac troponin levels when acute coronary syndromes are excluded. Ann Intern Med 2005; 142: 786-91. [CrossRef]

20. Fesmire FM, Christenson RH, Fody EP, Feintuch TA. Delta creatine kinase-MB outperforms myoglobin at two hours during the emergency department identification and exclusion of troponin positive non-STsegment elevation acute coronary syndromes. Ann Emerg Med 2004; 44: 12-9. [CrossRef] 